\title{
How useful is ARFI elastography for predicting the significant fibrosis and compensated liver cirrhosis?
}

\author{
Coskun Ozturker ${ }^{1}$, Ergenekon Karagoz ${ }^{2}$, Hakan Mutlu ${ }^{3}$
}

${ }^{1}$ Canakkale Military Hospital Department of Radiology, Canakkale, ${ }^{2}$ Van Military Hospital Department of Infectious Diseases, Van, ${ }^{3}$ GATA Haydarpasa Teaching Hospital Department of Radiology, Istanbul, Turkey

\section{To the Editor,}

In a recent issue of the Medical Ultrasonography we have read with great interest the article by Bota et al [1]. We would like to thank the authors for their comprehensive contribution. However, we would like to report few concerns regarding this study from a methodological point of view.

First, the measurements of ARFI were performed in the right liver lobe through the intercostal space [1]. Uslu et al demonstrated that subcostal approach to the liver parenchyma was significantly superior to intercostal approach for the evaluation of stiffness in their study [2]. It is also crucial to state that there is heterogeneity in the whole liver regarding the fibrotic distribution. Different fibrotic stages may be measured in different segments of liver either by liver biopsy or non-invasive methods. In this point of view, it would have been more relevant if the authors had assessed the liver fibrosis levels of the

Received 15.11.2015 Accepted 14.12.2015

Med Ultrason

2016, Vol. 18, No 1, 131, DOI: 10.11152/mu.2013.2066.181.coz

Corresponding author: Dr. Coskun Ozturker

Department of Radiology,

Canakkale Military Hospital,

Canakkale, Turkey

Phone: +905071425910

E-mail: drozturker@gmail.com patients according to different liver segments and noted the distribution of the measurements of these segments.

Second, in the present study, it was stated that all liver specimens obtained by liver biopsy were at least $2 \mathrm{~cm}$ long. However, it was demonstrated in most of the trials that a $25 \mathrm{~mm}$ long biopsy was considered an optimal specimen for accurate evaluation of liver fibrosis [3]. It would have been relevant, if the authors had mentioned these conditions as limitations.

We think that ARFI elastography may be a useful method for diagnosing the liver cirrhosis. Further studies are needed to indicate the role of ARFI method in the management of liver fibrosis level and cirrhosis.

\section{References}

1. Bota S, Sporea I, Sirli R, et al. How useful are ARFI elastography cut-off values proposed by meta-analysis for predicting the significant fibrosis and compensated liver cirrhosis? Med Ultrason 2015; 17: 200-205.

2. Uslu A, Batur A, Biy1k M, et al. Non-invasive evaluation of liver fibrosis using real-time elastography and comparison of intercostal and subcostal approaches. Eur J Gen Med 2015; 12: 109-113.

3. Bedossa P, Dargere D, Paradis V. Sampling variability of liver fibrosis in chronic hepatitis C. Hepatology 2003; 38: 1449-1457.

\section{Author's response}

\section{Simona Bota}

Department of Gastroenterology and Hepatology, "Victor Babeș” University of Medicine and Pharmacy, Timișoara, Romania

Firstly we want to thank for the interest in our article regarding how useful is ARFI elastography for predicting the presence of significant fibrosis and compensated liver cirrhosis [1]. 
Regarding our paper were raised the concerns regarding the measurements of liver stiffness by ARFI elastography through intercostal approach. Different from Uslu et al [2], we used this approach which is also recommended by the elastography guidelines of the European Federation of Societies for Ultrasound in Medicine and Biology (EFSUMB) [3].

Regarding the place for liver stiffness determination by ARFI elastography, we previously published a study [4], in which we showed that the ARFI values obtained in segment V and VIII of the liver were similar. The other published studies performed also the ARFI measurements only in a single point from the right liver lobe. The published data demonstrated only that the liver stiffness values from left liver lobe are significantly higher in comparison with the values obtained in the right lobe and from this reason it is recommended to perform the measurements only in the right lobe [5].

Regarding the quality of liver specimens, we included in the study specimens with at least $2 \mathrm{~cm}$ in length. This is not an limitation in our opinion, because the quality of biopsies was very good in our study. The median length of liver specimens was $3.5 \mathrm{~cm}$, raging from 2 to $6 \mathrm{~cm}$. We have to specify that only in $9 \%$ of the patients the length of biopsy specimen was between 2-2.5 $\mathrm{cm}$ and in $52.2 \%$ of the patients the specimen had at least $3.5 \mathrm{~cm}$ in length.
We strongly believe that ARFI elastography is a good technique for non-invasive assessment of liver fibrosis, as we demonstrated in a published meta-analysis [6].

\section{References}

1. Bota S, Sporea I, Sirli R, et al. How useful are ARFI elastography cut-off values proposed by meta-analysis for predicting the significant fibrosis and compensated liver cirrhosis? Med Ultrason. 2015; 17: 200-5.

2. Uslu A, Batur A, Biyık M, et al. Non-Invasive Evaluation of Liver Fibrosis Using Real-Time Elastography and Comparison of Intercostal and Subcostal Approaches. Eur J Gen Med 2015; 12: 109-113.

3. Cosgrove D, Piscaglia F, Bamber J, et al. EFSUMB guidelines and recommendations on the clinical use of ultrasound elastography. Part 2: Clinical applications. Ultraschall Med. 2013; 34: 238-53.

4. Bota S, Sporea I, Sirli R, et al. Factors that influence the correlation of acoustic radiation force impulse (ARFI), elastography with liver fibrosis. Med Ultrason. 2011; 13: 135-40.

5. Bota S, Peck-Radosavljevic M. Non-invasive evaluation of patients with viral hepatitis. Minerva Gastroenterol Dietol 2014; 60: 39-54.

6. Bota S, Herkner H, Sporea I, et al. Meta-analysis: ARFI elastography versus transient elastography for the evaluation of liver fibrosis. Liver Int. 2013; 33: 1138-47.

\section{Usefulness of ultrasound for the diagnosis of dermatofibroma}

\section{Kian Zarchi ${ }^{1}$, Charles B. Kromann ${ }^{1}$, Ximena Wortsman², Gregor B.E. Jemec ${ }^{1}$}

${ }^{1}$ Department of Dermatology, Roskilde Hospital, Faculty of Health and Medical Sciences, University of Copenhagen, Denmark, ${ }^{2}$ Department of Radiology and Department of Dermatology, Institute for Diagnostic Imaging and Research of the Skin and Soft Tissues, Clinica Servet, Faculty of Medicine, University of Chile, Santiago, Chile

Dermatofibromas are common benign skin tumors that are readily recognized by dermatologists in most cases. Nonetheless, they sometimes mimic other cuta-

Received 25.10.2015 Accepted 08.11.2015

Med Ultrason

2016, Vol. 18, No 1, 132-133, DOI: 10.11152/mu.2013.2066.181.kza

Corresponding author: Kian Zarchi,

Department of Dermatology, Roskilde Hospital,

Koegevej 7-13, 4000, Roskilde, Denmark

Phone: +4547322600

Fax number: +4547322698

E-mail: kian_zarchi@yahoo.com neous tumors, thus occasionally presenting a diagnostic challenge [1]. It is suggested that in clinically doubtful cases, ultrasound examination can provide clinicians with valuable diagnostic information [2].

A 34-year-old woman was referred with a skin tumor on her right upper arm. The tumor had been noticed one year previously by the patient. The lesion was stable, and the only complaint was occasional itching at the tumor site. The patient did not recall any preceding trauma or history of malignant skin tumors. During the clinical examination, a single lesion consisting of a 


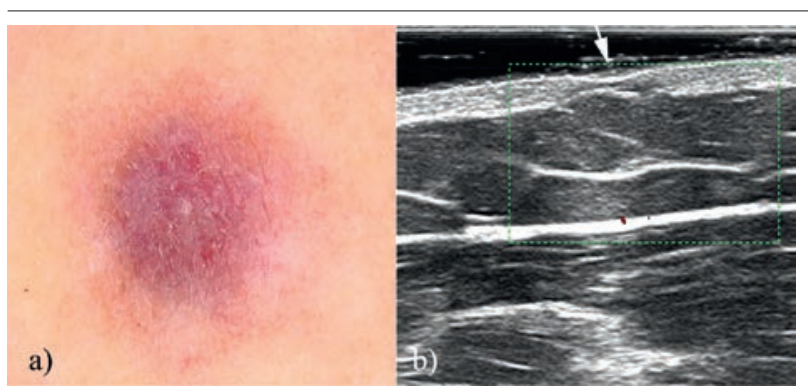

Fig 1. a) A symmetrical round tumor with a purple-red center, delicate pigment network, and scar-like tissue at its periphery; b) Color Doppler ultrasound revealing an ill-defined, hypoechogenic, atrophic lesion in the dermis and a lack of vascularity within and around the tumor.

slightly depressed, round tumor, measuring $5 \mathrm{~mm}$ in diameter was identified on the proximal part of the upper arm. Dermoscopic examination revealed a symmetrical round tumor with an irregular-surfaced purple-red center and with delicate pigment network and scar-like tissue at its periphery (fig 1a). Based on the clinical examination, a vascular tumor or a basal cell carcinoma was suspected. To explore the vascularity of the tumor, an ultrasound examination was performed using a MyLab Twice equipment (Esaote, Genova, Italy) with a broadband linear probe with variable frequency from 6 to $18 \mathrm{MHz}$. The examination revealed an ill-defined hypoechogenic focal lesion in the dermis and thinning of the dermis at the lesional site; color Doppler examination showed no signs of vascularity within or around the tumor (fig 1b). The ultrasound examination ruled out a vascular tumor and was not indicative of a basal cell carcinoma, as the sonographic characteristics of a basal cell carcinoma include a hypoechogenic, solid tumor with irregular borders, multiple hyperechogenic dots inside the tumor and vessels inside and at the periphery of the tumor [3]. Dermatofibromas, on the other hand, appear on ultrasound as an ill-defined or oval shaped hypoechogenic tumor in the dermis and/or upper hypodermis, usually with distortion of the local hair follicles $[2,4]$; accordingly, a tentative diagnosis of an atrophic dermatofibroma was made. The sonographic diagnosis was subsequently confirmed by a histological examination.

Dermatofibroma (fibrous histiocytoma) is a common dermal skin lesion characterized by an increased number of fibrocytes, presence of inflammatory cells (macrophages and lymphocytes), and hyperplasia of adjacent structures. Dermatofibroma has many clinical variants, including aneurysmal, palisading, sclerotic, keloidal, and atrophic and is associated with various dermatoscopic patterns, making clinical diagnosis difficult. A retrospective study of 122 histologically-verified dermatofibromas revealed that every fifth dermatofibroma was not correctly identified by a dermatologist [5]. The high-frequency ultrasound technique has been a valuable addition to the diagnostic toolbox of dermatologists. It allows a real-time view of the skin with discrimination of structures that measure $\geq 0.1 \mathrm{~mm}$ and provide highly useful information on the blood flow in the skin and its surroundings [2].

This case illustrates the clinical usefulness of ultrasound examination in difficult and doubtful cases of skin tumors.

\section{References}

1. Calonje E, Mentzel T, Fletcher CD. Cellular benign fibrous histiocytoma. Clinicopathologic analysis of 74 cases of a distinctive variant of cutaneous fibrous histiocytoma with frequent recurrence. Am J Surg Pathol 1994;18: 668-676.

2. Wortsman X, Wortsman J. Clinical usefulness of variablefrequency ultrasound in localized lesions of the skin. J Am Acad Dermatol 2010; 62: 247-256.

3. Wortsman X, Vergara P, Castro A, et al. Ultrasound as predictor of histologic subtypes linked to recurrence in basal cell carcinoma of the skin. J Eur Acad Dermatol Venereol 2015; 29: 702-707.

4. Wortsman X, Bouer M. Common benign non-vascular skin tumors.In: Dermatologic Ultrasound with Clinical and Histologic Correlations, Wortsman X, Jemec GBE eds, 1st edition, Springer NY 2013: 119-175

5. Han TY, Chang HS, Lee JH, Lee WM, Son SJ. A clinical and histopathological study of 122 cases of dermatofibroma (benign fibrous histiocytoma). Ann Dermatol 2011; 23: 185-192. 


\title{
Refractory knee synovitis due to suprapatellar plica persistence - the role of ultrasonography in diagnosis and management
}

\author{
Violeta Bojinca ${ }^{1}$, Raluca Malitchi ${ }^{1}$, Mihaela Micu ${ }^{2}$
}

${ }^{1}$ Department of Internal Medicine and Rheumatology, "Sf Maria" Hospital, Bucharest, ${ }^{2}$ Rheumatology Division, Department of Rehabilitation II, Rehabilitation Clinical Hospital, Cluj-Napoca, Romania

\section{To the Editor,}

We present the case of a 40 years old woman visiting our rheumatology department, complaining of chronic, inflammatory right knee pain and swelling associated with reduced range of motion refractory to analgesic, NSAIDs drug administration, and intra-articular corticosteroid injection (blind maneuver technique). She just started step-aerobic exercises 4 months ago but had to give up rapidly because of the right knee pain. Clinical examination revealed suprapatellar bursitis and limited knee flexion and extension. Conventional X- ray and routine blood laboratory analysis were in normal range. Musculoskeletal ultrasound (MSUS) of the right knee identified the persistence of the suprapatellar plica associated with synovial hypertrophy and effusion in both compartments - articular superior recess and suprapatellar bursa (fig 1a). MSUS guided puncture and aspiration in both compartments (articular recess and suprapatellar bursa) was performed using two separate needles and synovial fluid analysis was realized (fig 1b-c). Septic and microcrystalline etiology was not confirmed. Intraarticular MSUS guided corticosteroid injection was performed separately at level of the lateral recess and the suprapatellar bursa. Follow-up evaluation at 72 hours revealed a dramatic decrease of the knee pain level and complete recovery of the knee extension. No additional painkillers were necessary.

Received 01.12.2015 Accepted 10.12.2015

Med Ultrason

2016, Vol. 18, No 1, 134-135, DOI: 10.11152/mu.2013.2066.181.rfc

Corresponding author: Violeta Bojinca

Clinical Hospital Sf. Maria

37-39 Ion Mihalache Bd

Bucharest, Romania

Fax: +040212223555

Email: vmbojinca@yahoo.com
The synovial plicae are normal synovial structures of the knee joint cavity, remnants of the mesenchymal tissue filling the space situated between the distal femoral and proximal tibial epiphyses in the 8-week-old embryo. The knee joint turns into one single cavity during the fourth to fifth fetal life month, by disappearance of the dividing septa that form the medial, lateral and supra-patellar compartments [1]. There were described four types of plicae of the knee [1-4]: suprapatellar (that rarely may initiate a suprapatellar bursitis or chondromalacia), infrapatellar (more commonly known as the ligamentum mucosum), mediopatellar (considered the most frequently cause of plica syndrome and sometimes known as a shelf), and lateral.

Generally these plicae are asymptomatic, but, in certain situations (like the presence of an exuberant local shelf at baseline and an inciting event - macrotrauma or repeated microtrauma) the patients might develop symp-

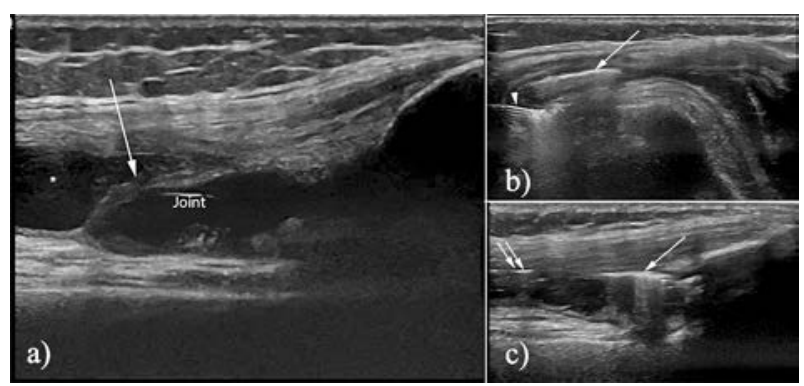

Fig1. a) Longitudinal suprapatellar scanning of the knee. Arrow - plica persistence, ${ }^{*}$ hypoechooic material (effusion) inside the suprapatellar bursa. Joint - effusion inside the joint recess; b) Transversal scanning at the level of the lateral para-patellar recess. Corticosteroid injection inside the lateral joint recess. Arrow - hyperechoic glucocorticoid deposition, arrow headneedle penetrating the lateral recess; c) Longitudinal suprapatellar scanning of the knee. Arrow - corticosteroid deposition inside the joint recess, double arrow- corticosteroid deposition inside the suprapatellar bursa. 
toms $[3,4]$. The history of physical activity or trauma is absent only in small number of patients. In the presence of inflammation, the normal local tissue may turn into truly pathologic structure, with edema, hypertrophy, and decreased elasticity. The pathologic plicae are described as thick, fibrotic, white, and inelastic, with fibrosis, hyalinization and calcification [4].

The clinical suspicion of the plica syndrome diagnosis represents a true challenge and may be confirmed by arthroscopy, MSUS, computer tomography with arthrography, and magnetic resonance imaging $[2,4,5]$.

MSUS may be a useful diagnostic tool for the identification of synovial plica differentiating it from other mimicking conditions. MSUS can be performed rapidly after the clinical examination, is a non-radiant and relative inexpensive imaging method. It allows the guiding of interventional maneuvers followed by precise drug deposition in both compartments and in this way having higher treatment efficacy [6].

\section{References}

1. Kent M, Khanduja V. Synovial plicae around the knee. Knee 2010; 17:97-102.

2. Kim SJ, Choe WS. Arthroscopic findings of the synovial plicae of the knee. Arthroscopy 1997; 13: 33-41

3. Bellary SS, Lynch G, Housman B, et al. Medial plica syndrome: a review of the literature. Clin Anat 2012; 25: 423428.

4. Schindler OS. The Sneaky Plica' revisited: morphology, pathophysiology and treatment of synovial plicae of the knee. Knee Surg Sports Traumatol Arthrosc 2014; 22: 247262.

5. Shetty VD, Vowler SL, Krishnamurthy S, Halliday AE. Clinical diagnosis of medial plica syndrome of the knee: a prospective study. J Knee Surg 2007; 20: 277-80.

6. Epis O, Bruschi E. Interventional ultrasound: a critical overview on ultrasound- guided injections and biopsies. Clin Exp Rheumatol 2014; 32(Suppl 80): S78-84.

\title{
Cesarean scar pregnancy: role of serial transabdominal ultrasonography in the diagnosis and treatment response following dilation and curettage.
}

\author{
Turker Acar ${ }^{1}$, Ali Cankat Sahin ${ }^{1}$, İsmail Semiz ${ }^{1}$, Bekir Gulac ${ }^{2}$
}

${ }^{1}$ Department of Radiology, ${ }^{2}$ Department of Obstetric and Gynecology Abant Izzet Baysal University Izzet Baysal Education and Training Hospital, Bolu, Turkey

\section{To the Editor,}

It was with great interest that we read the paper by Elito Júnior et al [1] and the related papers regarding cesarean scar pregnancy (CSP) in Medical Ultrasonography. CSP is an extremily rare type of ectopic pregnancy which may result with uterine rupture or life-threatening hemorrhage if diagnosed at later stages. Moreover,

Received 27.10.2015 Accepted 08.11.2015

Med Ultrason

2016, Vol. 18, No 1, 135-136, DOI: 10.11152/mu.2013.2066.181.tua

Corresponding author: Turker Acar

Department of Radiology,

Abant Izzet Baysal University

Izzet Baysal Education and Training Hospital

Sağlık Mh. Şehitler Cad., 14300 Bolu, Turkey

Phone: +90 (374) 2704575

Fax: +90 (374) 2534615

E-mail: drtacar@hotmail.com these potential complications may lead hysterectomy and cause dramatic consequences for future reproductive capacity. The treatments for CSP include regular dilation and curettage (D\&C) alone, methotrexate (MTX) alone or in conjunction with bilateral uterus artery embolization (UAE), or D\&C suction after UAE [1]. Transvaginal ultrasonography (TVUS) is the cornerstone imaging modality in the diagnosis of CSP [2]. Magnetic resonance imaging (MRI) is a problem solver particularly when sonographic findings are equivocal or inadequate before planning intervention [3]. Due to the fact that TVUS is not always technically possible in the radiology departments of secondary care units, familiarity with the transabdominal US features of CSP is critical for the diagnosis. According to best of our knowledge, literature related with early sonographic chances after D\&C and long term follow-up imaging findings are limited. In this 


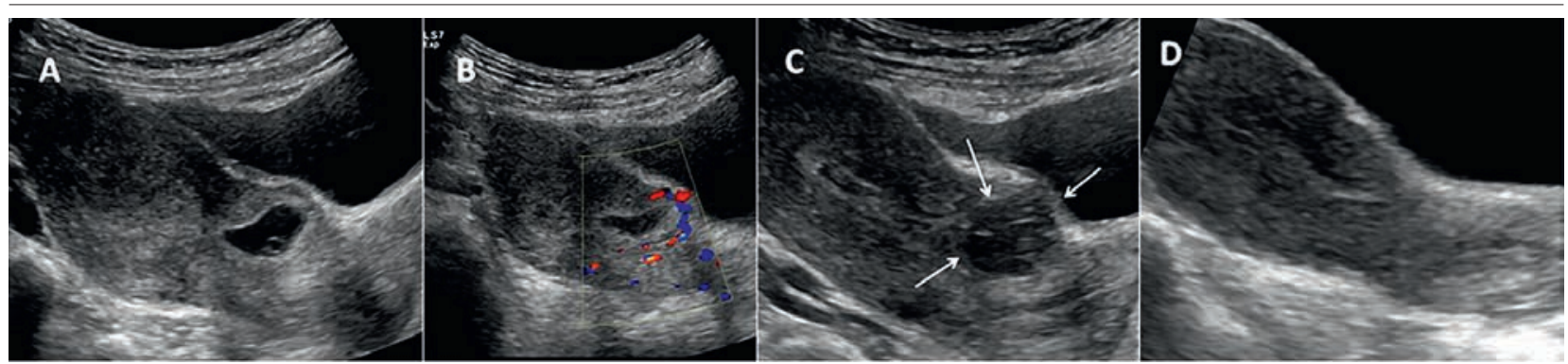

Fig 1. Sagittal grey scale (A), and Doppler ultrasound images (B) showing the gestational sac in the cesarean scar. Peritrophoblastic rim like marked vascularity is seen. Note that bladder and serosal surface of the uterus is intact. Sagittal ultrasound image after D\&C shows (C) coagulum in the gestational sac and distruption of Yolk sac (arrows). Ultrasound image obtained on the last visit (D) shows full recovery.

letter, we aimed to share our experience in a woman diagnosed with CSP who was managed with systemic MTX treatment following D\&C and underline potential role of repetitive transabdominal US in the treatment response with demonstrative images.

A 31 year-old uniparous woman with a prior caesarean history was admitted to obstetrics department of our institution with menstruation delay and vaginal bleeding started 4 days ago. She was hemodynamically stable with minimal bleeding during speculum examination. Beta fraction of human chorionic gonadotropin (B-HCG) was $3,995 \mathrm{mIU} / \mathrm{mL}$ in the initial submission and TVUS revealed ectopic pregnancy in the old caesarean scar. In the second visit, B-HCG level elevated to 7,627 mIU/ $\mathrm{mL}$ accompanied with increased gestational sac diameters. Transabdominal gray scale, Doppler US (Fig 1A, 1B) confirmed the diagnosis. Firsty, she was treated with single dose MTX which was well tolerated by the patient. Despite medical treatment, B-HCG levels rised gradually in the following visits and a complementary D\&C was performed. US images obtained immediately after D\&C showed total obliteration of GS and distruption of YS replaced with coagulum (Fig 1C). The procedure was completed without complication and she was discharged in the same day. B-HCG levels dropped gradually to 0 within 2 weeks. US evaluation of cesarean scar and uterine integrity returned to normal limits in the last visit obtained after 50 days of D\&C (Fig 1D).

In conclusion, several treatment methods have been reported for the management of CSP. Although early transvaginal sonography is the reference standard for diagnosis in the first trimester, transabdominal US (if performed under the optimal conditions) has a potential adjunct not only in the diagnosis, but also in the monitorization of treatment response in patients who underwent dilation and curettage.

\section{References}

1. Elito Júnior J, Araujo Júnior E, Martins Santana EF, et al. Uterine artery embolization with methotrexate infusion as treatment for cesarean scar pregnancy. Case report. Med Ultrason 2013; 15: 240-243.

2. McKenna DA, Poder L, Goldman M, Goldstein RB. Role of sonography in the recognition, assessment, and treatment of cesarean scar ectopic pregnancies. J Ultrasound Med 2008; 27: 779-783.

3. Riaz RM, Williams TR, Craig BM, Myers DT. Cesarean scar ectopic pregnancy: imaging features, current treatment options, and clinical outcomes. Abdom Imaging 2015; 40: 2589-2599. 